
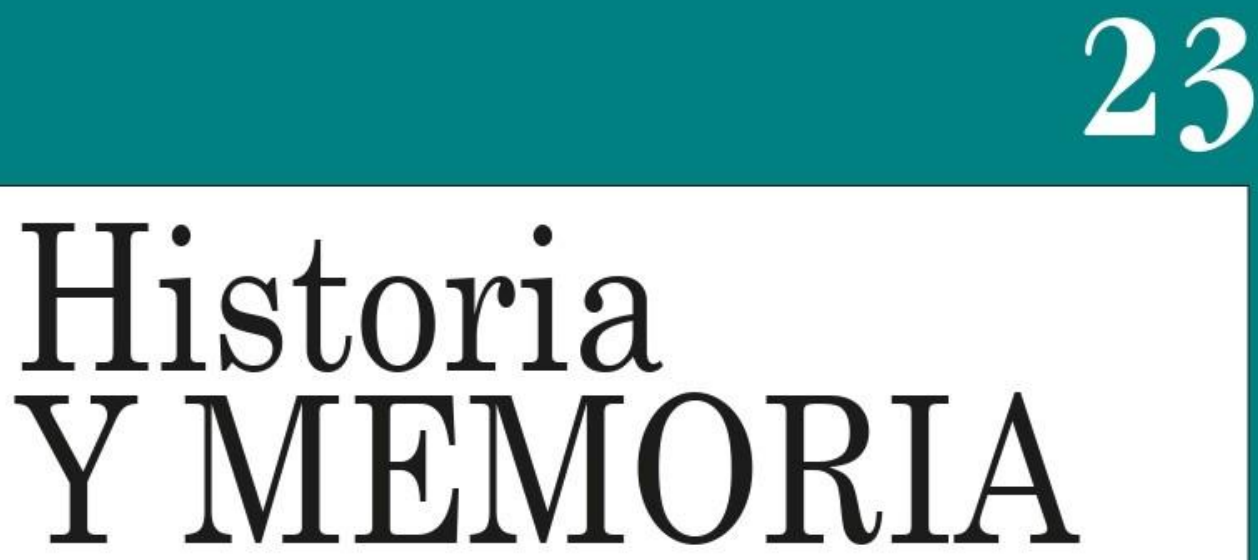

ISSN: 2027-5137 Julio - Diciembre, Año 2021 - Tunja, Colombia

Maternidad y lactancia a través del discurso de la comunidad médica en Barranquilla, primera mitad del siglo XX

https:/doi.org/10.19053/20275137.n23.2021.11720

Ángela Lucía Agudelo-González Willian Alfredo Chapman-Quevedo Páginas 197-225

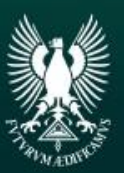




\title{
Maternidad y lactancia a través del discurso de la comunidad médica en Barranquilla, primera mitad del siglo $\mathrm{XX}^{*}$
}

\author{
Ángela Lucía Agudelo-González ${ }^{1}$ \\ Willian Alfredo Chapman-Quevedo ${ }^{2}$ \\ Universidad del Tolima - Colombia
}

Recepción: 09/09/2020

Evaluación: 28/03/2021

Aprobación: 25/04/2021

Artículo de Investigación e Innovación

https:/doi.org/10.19053/20275137.n23.2021.11720

\begin{abstract}
* Resultado del proyecto de investigación: Salubridad, higiene y criminalidad en los departamentos de Atlántico y Caldas durante la primera mitad del siglo XX. Financiado por la Universidad del Atlántico y la Universidad del Tolima (código interno: 100115); también es parte del proyecto de tesis doctoral de la profesora Ángela Lucía Agudelo González del programa de Historia y Artes de la Universidad de Granada titulado: «Salubridad pública y Mujer en las ciudades de Barranquilla e Ibagué entre 1900-1950». 1 Profesora Asociada del Departamento de Psicopedagogía de la Facultad de Ciencias de la Educación de la Universidad del Tolima. Doctoranda del programa de Historia y Artes de la Universidad de Granada (España) y Magíster en Geografía (Universidad de los Andes); Historiadora (Universidad del Atlántico). Entre sus últimas publicaciones están: en coautoría, «Transformando el país a través de la Higiene. Las cartillas técnicas y la revista Rin Rin en las bibliotecas aldeanas de Colombia 1936-1938», Historia y Espacio vol. 16, n ${ }^{\circ} 54$ (2020), doi: https://doi.org/10.25100/hye.v16i54.9665; en coautoría, «La prostitución como un problema de salubridad pública: Prostitutas, violencia y leyes en el Departamento del Atlántico, 1911-1950», Historia Caribe vol. 15, nº 37 (2020), doi: https://doi.org/10.15648/hc.37.2020.8, e «Imagen de ciudad. Las fotografías publicadas en el Directorio Comercial Pro-Barranquilla y el Álbum de Cartagena de Indias durante la década del 20 del siglo XX», en Colombia: Historia, Educación y Política Miradas Múltiples, ed. Luis Alarcón Meneses (Barranquilla: Universidad del Atlántico, 2019) 247272.『 alagudelog@ut.edu.co (1) https://orcid.org/0000-0001-7747-3517.

2 Profesor Asociado del Departamento de Psicopedagogía de la Facultad de Ciencias de la Educación de la Universidad del Tolima. Doctor en Historia Social y Política Contemporánea: Movimientos Sociales y Construcción de la Ciudadanía en el Mundo Contemporáneo en Perspectiva Comparada (Universidad Internacional de Andalucía), Magíster en Sociedades Históricas y Formas Políticas en Europa (Universidad Rovira i Virgili), Historiador (Universidad del Atlántico). Entre sus últimas publicaciones están: en coautoría, «Trasformando el país a través de la Higiene. Las cartillas técnicas y la revista Rin Rin en las bibliotecas aldeanas de Colombia 1936-1938», Historia y Espacio vol. 16, $\mathrm{n}^{\circ} 54$ (2020), doi: https://doi.org/10.25100/hye.v16i54.9665; en coautoría, «La prostitución como un problema de salubridad pública: Prostitutas, violencia y leyes en el Departamento del Atlántico, 1911-1950», Historia Caribe vol. 15, no 37 (2020), doi: https:// doi.org/10.15648/hc.37.2020.8; en coautoría, «De amateurs a profesionales. Una historia social del fútbol en Barranquilla durante la primera mitad del siglo XX», en Fútbol: una mirada interdisciplinar. Ocio, literatura, educación, historia y ética (Ibagué: Universidad del Tolima, 2020), 73-102. $₫$ wachapmanq@ut.edu.co 『 https://orcid.org/0000-0003-24151535 .
\end{abstract}




\title{
Resumen
}

En este artículo se analiza el papel de la maternidad y de la lactancia, a través de los cánones y consejos de los médicos difundidos en los medios de comunicación de la primera mitad del siglo XX. Mostramos, en primer lugar, que la madre jugaba un rol importante en la sociedad, ya que se concibió como el eje de la transformación y el progreso de Colombia, y, en segundo lugar, cómo a través de los discursos médicos se intentó transformar las prácticas alrededor de la lactancia. La metodología trazada incluyó el análisis de fuentes primarias (columnas, conferencias y manuales) y secundarias (artículos y libros sobre la temática). La conclusión de esta investigación afirma que existió una preocupación por parte de la comunidad médica en transformar la maternidad en un asunto público bajo políticas de higiene y salubridad que ayudaran al proceso de modernización del país.

Palabras claves: Maternidad, lactancia, salud, mujer, salubridad pública.

\section{Maternity and breastfeeding through the discourse of the medical community in Barranquilla in first half of the 20 th century}

\begin{abstract}
This article analyses the role of maternity and breastfeeding through the canons and advice of doctors, circulated in mass media in the first half of the 20th century. It is shown, firstly, that the mother played an important role in society, as she was viewed as the axis of the transformation and progress of Colombia, and, secondly, how an attempt was made to transform the practices around breastfeeding through medical discourses. The methodology used included the analysis of primary sources (columns, conferences, and manuals) and secondary sources (articles and books on the topic). The conclusion of this investigation affirms that there was an interest on the part of the medical community ink transforming maternity into a public matter under policies of
\end{abstract}


hygiene and health that would help the modernization process of the country.

Key words: Maternity, breastfeeding, health, women, public health.

\section{La maternité et l'allaitement dans le discours de la communauté médicale de Barranquilla dans la première moitié $\mathrm{du} \mathrm{XX}^{\mathrm{e}}$ siècle}

\section{Résumé}

Cet article analyse le rôle que la maternité et de l'allaitement avaient dans les canons et les conseils des médecins popularisés par les mass-média dans la première moitié du $\mathrm{XX}^{\mathrm{e}}$ siècle. On tente de montrer, d'abord, que la mère jouait un rôle important dans la société, car elle était conçue comme l'axe principal de la transformation et du progrès de la Colombie ; puis, on revient sur les manières dont les discours médicaux cherchaient à transformer les pratiques liées à l'allaitement. La méthodologie utilisée consiste à analyser des sources primaires telles que tribunes, conférences et manuels ainsi que des sources secondaires, notamment articles et livres sur le sujet. En guise de conclusion, on propose qu'il a existé un effort chez la communauté médicale pour transformer la maternité en une affaire publique qui, dans le cadre de l'hygiène et la salubrité, serait censée contribuer au processus de modernisation du pays.

Mots-clés: Maternité, allaitement, santé, femme, salubrité publique.

\section{Introducción}

A finales del siglo XIX y principios del XX se empezó a desarrollar en Colombia una corriente médica que buscó mejorar las condiciones de salubridad pública, influenciada por la reciente asimilación de las disposiciones de la medicina 
pasteuriana $^{3}$. Este tipo de medicina se caracterizó por combinar el estudio de las enfermedades con una mejora en las condiciones de vida de la población ${ }^{4}$. Su principal eje de actuación se concentró en las condiciones de vida porque veían en esto el foco de propagación de las enfermedades.

A partir de esta preocupación por la población se empezó a generar toda una serie de discursos alrededor de la maternidad, con el propósito de encausar los hábitos de higiene de las madres y a través de estos actuar sobre la niñez, considerada durante el período eje fundamental para la modernización de la nación. Esto trajo consigo una doble configuración, así, entre mayor era la importancia que se le daba a la infancia más relevante fue el papel de la madre ${ }^{5}$.

Este interés por el rol materno, en que el papel de la mujer estaba inmerso en los cambios que vivía el país en ese momento, destacándose la inserción de la mujer en el mundo laboral y el surgimiento de nuevas ocupaciones laborales ${ }^{6}$, implicando un cuestionamiento al modelo de familia católica y nuclear ${ }^{7}$. Así mismo, las mujeres consolidaron su entrada a la esfera pública abriéndose un lugar en el ámbito político, incluso mucho antes de que obtuviera el derecho al voto ${ }^{8}$, reflejado en su participación en los procesos de cuidado de

3 Robert Bud, Penicillin Triumph and Traged (New York: Oxford University Press, 2007), 54-75; Jorge Márquez Valderrama, Ciudad, miasmas y microbios. La irrupción de la ciencia pasteriana en Antioquia (Medellín: Universidad de Antioquia, 2005), 24-71.

4 Luis Urteaga, «Higienismo y ambientalismo en la médicina decimononica», Acta Hispánica ad Medicinae Scientiarumque Historiam Illustrandam, vol. 5-6, (1985): 417-425.

5 Cristina Palomar Verea, "Maternidad: Historia y cultura», La ventana, $\mathrm{n}^{\circ} 22$ (2005): 35-66.

6 Ana Catalina Reyes «Mujeres y trabajo en Antioquia durante la primera mitad del siglo XX», en Mujer, nación, identidad y ciudadanía: siglos XIX y XX. IX Cátedra Anual de Historia Ernesto Restrepo Tirado (28 al 30 de octubre de 2004), ed. Ana María Díaz Granados (Bogotá: Museo Nacional, 2005), 162-192.

7 Suzy Bermúdez, El Bello sexo, la mujer y la familia durante el Olimpo radical (Bogotá: Ediciones Uniandes, ECOE ediciones, 1993), 70.

8 María Fátima Barceló, «Domesticidad, desafío y subversión: la discursividad femenina», Óp. cit. Revista del centro de investigaciones históricas, $\mathrm{n}^{\circ} 14$ (2002): 187 -212 . 
los desfavorecidos que tuvo injerencia en las decisiones de la política local barranquillera ${ }^{9}$.

Estas transformaciones llevaron al desarrollo de un nuevo tipo de maternidad que se empezó a desarrollar por fuera del espacio doméstico ${ }^{10}$. Esto trajo consigo nuevas miradas al rol asignado por la sociedad a la mujer como madre, esposa y primera educadora encargada de la vida doméstica. Fue así como en una serie de publicaciones realizadas por los médicos de la época, empezó a llamar la atención sobre la importancia de que la mujer asumiera los roles tradicionales asignados.

Tomando como punto de partida estos elementos, el presente artículo busca entender que la maternidad no puede ser observada y estudiada como un proceso atemporal, sino que la misma ha sido configurada según el contexto histórico en el cual se desarrolla. Es así como:

La maternidad no es un "hecho natural", sino una construcción cultural multideterminada, definida y organizada por normas que se desprenden de las necesidades de un grupo social específico y de una época definida de una historia. Se trata de un fenómeno compuesto por discursos y prácticas sociales que conforman un imaginario complejo y poderoso que es, a la vez fuente y efecto del género ${ }^{11}$.

En este sentido, en el presente artículo buscamos entender el papel de la maternidad por medio de los cánones y consejos de la comunidad médica de Barranquilla en la primera mitad del siglo XX. Para lograrlo, nos trazamos una ruta metodológica que incluyó la revisión de distintas publicaciones seriadas como la Revista Departamental de Higiene del Atlántico y el periódico El Tiempo (con alcance nacional), al igual que las conferencias en formato de audio que reposan en la Biblioteca Nacional y algunas transcritas e impresas en los periódicos y revistas de la época, y por

9 Rafaela Vos Obeso, Mujer, cultura y sociedad: Barranquilla, 1900-1930 (Santa Fe de Bogotá: Fondo de Publicaciones de la Universidad del Atlántico, 1999), 320.

10 Amparo Micolta, "Apuntes históricos de la paternidad y la maternidad», Prospectiva, $\mathrm{n}^{\circ} 13$ (2008): 105.

11 Palomar, «Maternidad: Historia y cultura...», 36. 
supuesto los manuales de puericultura traducidos o realizados durante el período estudiado. Así mismo, nos apoyamos en los estudios sobre la maternidad, la mujer, la higiene y la salubridad producidos por la historiografía contemporánea. Esto nos permitió identificar cómo fue que se construyó la idea sobre la maternidad y el papel de la lactancia durante el período estudiado.

El artículo está dividido en dos partes: en la primera observamos cómo los discursos médicos se centraron en el rol de las madres y en el cuidado que debían brindar a sus hijos en búsqueda de trasformar sus comportamientos y prácticas. En la segunda, atendemos la posición médica relacionada con la nutrición infantil, específicamente la lactancia y el uso de la alimentación láctea en que los médicos generaron consejos y opiniones, provocando controversias entre el conocimiento científico y el tradicional femenino.

\section{El papel de la maternidad en los discursos médicos}

Durante la primera mitad del siglo XX circularon en Colombia una serie de escritos, cartillas, columnas, manuales y audios, cuyos autores eran médicos donde mostraban una preocupación sobre la maternidad como eje de medicalización ${ }^{12}$, mediante los cuales el sector sanitario buscó dar solución a las exigencias de la sociedad colombiana. En estos escritos se comenzó a concebir a la madre como «el molde sacro de la raza y de cuya pericia depende, en grado sumo, el porvenir físico del niño» ${ }^{13}$.

Este fenómeno fue alimentado por las importantes transformaciones que se estaban dando en Colombia como lo fue la inserción de las mujeres al mercado laboral, alimentada por las migraciones internas de lo rural a lo urbano, lo

12 Entendemos la medicalización «como la conversión en procesos patológicos de situaciones que son y han sido siempre completamente normales y el pretender resolver mediante la medicina situaciones que no son médica sino sociales, profesionales o de las relaciones interpersonales» Ramón Sánchez y otros, «Medicalización de la vida (I)», Rev Clin Med Fam vol. 4, no 2 (2011): 151.

13 Calisto Torres Umaña y Eduardo Vasco Gutierrez, Nociones de Puericultura: El cuidado de la salud y la Educación del Cáracter (Bogotá: Imprenta Municipal, 1935), 1. 
que permitió que se aumentara la oferta de mano de obra accesible y barata, puesto que estas ganaban la mitad de lo que se les pagaba a los hombres ${ }^{14}$. Este tipo de mano de obra contribuyó al desarrollo industrial del país. Un ejemplo de lo afirmado anteriormente lo encontramos en el censo de $1928^{15}$, donde se refleja que en la ciudad de Barranquilla ya empezaban a aparecer y a tomar fuerza ocupaciones, más allá de los oficios domésticos, como lo eran las empleadas públicas, comerciantes ${ }^{16}$, tejedoras, costureras, modistas y mecanógrafas. Este proceso se consolidó con el impulso dado por la industria textil, donde la mano de obra femenina representaba casi el $80 \%$ de la fuerza laboral contratada durante las primeras décadas del siglo $\mathrm{XX}^{17}$.

En Colombia el empleo femenino ${ }^{18}$ se convirtió en una preocupación reflejándose en los artículos de prensa, publicados entre las décadas de los 30 y 40 del siglo XX, que recomendaban que «el empleo más importante» que podía ejercer una mujer era estar en su hogar y por ejemplo citaban las palabras de la esposa de Thomas Alva Edison, quien afirmaba:

Yo no veo nada servil, ni monótono, ni rutinario en la tarea que nos impone el manejo del hogar-asegura la esposa del famoso inventor americano, Thomas Alva Edison- Me parece

14 Daniela Santos Cárdenas, «Faldas por pantalones: las obreras, la brecha salarial y el sindicalismo femenino en la industria en Colombia en 1945», Documentos CEDE $\mathrm{n}^{\circ} 49$ (2017): 2; Reyes «Mujeres y trabajo...», 162-192.

15 «Boletín Municipal de Estadística», Barranquilla, 1930, Archivo Histórico del Atlántico (AHA), Barranquilla-Colombia. Fondo Colección Hemerográfica, No.1.

16 La inserción de la mujer al comercio en la ciudad de Barranquilla se puede rastrear desde la segunda mitad del siglo XIX. Ver: Tomás Caballero Truyol, «Entre los negocios y la familia: Mujer, crédito y sociedad en Barranquilla (Colombia), 18491900», Historelo vol. 9, nº 17 (2017): 197-220, doi: http://dx.doi.org/10.15446/historelo. v8n16.53942.

17 Santiago Montenegro, El arduo tránsito hacía la modernidad: historia de la industria textil colombiana durante la primera mitad del siglo XX (Medellín: Universidad de Antioquia; Norma, CEDE, 2002), 225-226.

18 En países como Estados Unidos la vinculación de la mujer en empleos como dependientas, maestras o en el ejercicio de profesiones liberales, al igual que su admisión en la universidad influyeron en la ampliación de la edad para contraer matrimonio. Nancy F. Cott, «Mujer moderna, estilo norteamericano: los años veinte», en Historia de las mujeres. El siglo XX, dir. George Duby y Michelle Perrot (Barcelona: Taurus, 1993), 107-124. 
llena de interés y una fuente de deleite. Comparada con ella, el empleo más importante que una mujer puede tener en una oficina resulta aburrido, maquinal y de estrechos límites ${ }^{19}$.

Sin embargo, los médicos colombianos tenían presente que era difícil que la mujer se quedara en casa, por lo tanto, proponían el desarrollo en los centros fabriles de salas cunas en donde los hijos de las obreras fueran cuidados. Así lo propuso el médico Luis Urdaneta, en 1930, el cual se inspiró en el modelo francés:

\begin{abstract}
[...] donde el trabajo femenino se ha difundido considerablemente, si no sería posible hacer ojalá algo semejante o a lo menos algo que, consultando nuestras necesidades y nuestro desarrollo fabril, diera resultados parecidos a los [de] estas instituciones que la conservación de la raza y el deber social requieren, se han obtenido en los países civilizados ${ }^{20}$.
\end{abstract}

Además de la inserción laboral de la mujer, otro elemento que preocupaba a la comunidad médica era la mortalidad infantil, sobre todo entre niños y niñas de 0 a 2 años. Por ejemplo, para el año de 1929 las cifras de mortalidad en Barranquilla en el rango de esta edad eran 53,18\% ${ }^{21}$. Como han mostrado varias investigaciones ${ }^{22}$ la causa de

19 «El empleo más importante», El Tiempo, Bogotá, 12 de enero de 1930, 12. Acceso el 3 de febrero de 2020, https://news.google.com/ newspapers?nid=N2osnxbUuuUC\&dat=19300112\&printsec=frontpage\&hl=es.

20 Doctor Luis A. Urdaneta, «la importancia de los consultorios y de la protección de las mujeres en cinta por el doctor Luis A. Urdaneta", El Tiempo, Bogotá, 22 de enero de 1930, 12. Acceso el 3 de febrero de 2020, https://news.google.com/ newspapers?nid=N2osnxbUuuUC\&dat=19300122\&printsec=frontpage\&hl=es.

21 "Boletín Municipal de Estadística», Barranquilla, 1932, Archivo Histórico del Atlántico (AHA), Barranquilla-Colombia. Fondo Colección Hemerográfica, No. 5, 14. Las cifras registradas para la ciudad de Barranquilla tienen una fuerte correlación con las del territorio colombiano en 1935, donde fallecieron 45,8 infantes de 0 a 2 años, los datos son tomados de: Oscar Gallo Velez y Jorge Marquez Valderrama, «La mortalidad infantil y la medicalización de la infancia. El caso de Titiribí, Antioquia, 1910-1950", Memoria y Sociedad, $\mathrm{n}^{\circ} 20$ (2011): 68. Otro caso es el presentado en Soledad (Atlántico) por el Secretario de Higiene y Asistencia Social del Departamento del Atlántico Arturo Ponce, quien acotaba las alarmantes cifras de mortalidad infantil de menores de cinco años que se encontraba en el 55\% de fallecido en 1943. Arturo Ponce, «El fondo de fomento municipal y la asistencia pública», Revista de Higiene, $n^{\circ} 29-30$ (1943): 9 .

22 Jorge Humberto Márquez Valderrama y Oscar Gallo Vélez, «Eufemismos para el hambre: saber médico, mortalidad infantil y desnutrición en Colombia, 1888-1940», 
la mortalidad infantil era originada principalmente por enfermedades infecciosas, respiratorias o gastrointestinales: enteritis, gastroenteritis, cólera y disentería, que combinadas con una pobre alimentación llevaron a la muerte a la población infantil. Esta situación fue expuesta por los medios de la época, quienes elevaron constantes quejas, como lo refleja un artículo publicado en la Revista de Higiene en 1944, en el cual se expresaban: "de mil niños que nacen, mueren por lo menos, doscientos por motivos de falta de buena nutrición de la madre. Estos niños mueren generalmente de diarreas, neumonías, acidosis» ${ }^{23}$.

Debido a la conjugación de ambos factores, la inserción femenina en el mercado laboral y los índices de mortalidad infantil, los médicos generaron serios cuestionamientos alrededor del instinto maternal de las mujeres, concebido como un don otorgado por la naturaleza ${ }^{24}$. A partir de esto se buscó mantener a la mujer en el hogar para que cumpliera con el rol social, que le fue asignado haciendo un llamado a través de la prensa para evitar que «desertarán las madres de las cunas, como hoy lo [hacían] obligadas por la necesidad y la injusticia» ${ }^{25}$.

Para lograrlo se aconsejaba seguir manteniendo las divisiones tradicionales de género: el hombre se ocupaba del

Historia y Sociedad, $\mathrm{n}^{\circ} 32$ (2017): 21-48, doi: https://doi.org/10.15446/hys.n32.55508; Gallo y Márquez, «La mortalidad infantil,» 68; Jessica Morales Rambaut, «Salubridad pública en Barranquilla en la decada de los treinta del siglo XX: principales causas de muerte y su inciencia con los hábitos de vida» (Tesis de pregrado, Universidad del Atlántico, 2013), 24.

23 Dr. Roberto Pardo, «Importancia del arroz en la alimentación del pueblo, charla del Doctor Roberto Pardo, Director del Departamento de nutrición del Servicio Cooperativo Interamericano de Salud Pública», Revista de higiene, $\mathrm{n}^{\circ} 35$ (1944): 28. También en El Tiempo se registró esta queja: «Todavía es una rémora para la capital la enorme sangre infantil con sus renglones de mortalidad por gastrointestinales y debilidad congénita; prevalecen elevados porcentajes algunas enfermedades infecto-contagiosas como la sífilis, la tuberculosis y la fiebre tifoidea» «Higiene y Saneamiento», El Tiempo, Bogotá, 26 de febrero de 1930, 12. Acceso el 05 de febrero 2020, https://news.google. $\mathrm{com} /$ newspapers?nid=N2osnxbUuuUC\&dat=19300226\&printsec=frontpage\&hl=es.

24 Micolta, "Apuntes históricos de la paternidad...», 106-107.

25 G. Martínez Sierra, «Nuevas cartas a las mujeres: La nueva ley que han de hacer las mujeres», El tiempo, Bogotá, 5 de enero 1930, 12. Acceso el 05 de febrero de 2020, https://news.google.com/ newspapers?nid=N2osnxbUuuUC\&dat=19300105\&printsec=frontpage\&hl=es. 
modo de producción y las mujeres del cuidado del hogar. Así lo afirmaba en 1930 el columnista Martínez «a los hombres nos incumbirán casi todos los trabajos de producción de medios de materiales para la vida; a las mujeres todos los de conservación y perfeccionamiento de la vida» ${ }^{26}$ y este rol maternal era prolongado en la vida de la mujer, ya que para él: «los hijos [eran] de ellas, porque la vida [era] de ellas, y los hombres siempre seremos un poco sus criaturas» ${ }^{27}$. Un proceso análogo se presentó en México durante la primera mitad del siglo XX, donde la conjugación de las ideas del positivismo, el liberalismo y la cristiandad llevaron a la adopción de una serie de discursos que impulsaban a la mujer a adoptar los papeles tradicionales de esposa y madre. Por lo tanto, "se requería de mujeres que permanecieran en el hogar, vigilaran la institución familiar y reprodujeran las relaciones sociales» ${ }^{28}$

De ahí que los médicos jugaron un papel importante en la propuesta de abogar por la conservación del rol tradicional de la mujer, ya que ellos establecieron los mecanismos necesarios para generar la intervención sobre la población femenina, buscando cumplir el fin social encomendado ${ }^{29}$, en el cual la maternidad era importante debido a que con esta se garantizaba el futuro de la nación, lo que llevó a que se difundiera la importancia de que todo bebé debía nacer y criarse en las mejores condiciones posibles ${ }^{30}$.

En este sentido, los médicos no escatimaron medios para difundir sus ideas y "por medio de la radio, hasta los últimos rincones de Barranquilla, la vulgarización de temas médicos [...] pueden interesar y ser provecho para todos los oyentes $»^{31}$,

26 Martínez, «Nuevas cartas...», 12.

27 Martínez, «Nuevas cartas...», 12.

28 Marcela Suárez, "La familia Burguesa y la mujer mexicana en los inicios del siglo XX», en Mujeres latinoamericanas del siglo XX. Historia y cultura Tomo II Luisa Campuzano (México: Universidad Autónoma metropolitana, Casa de las Américas, 1998), 295.

29 Marlene Sánchez, Representación de la mujer en Bogotá 1880-1920, Tomo II (Bogotá: Fundación para la promoción de la investigación y la tecnología, 1999), 196.

30 Micolta, «Apuntes históricos de la paternidad...», 102.

31 Dr. Ernesto Brando, «Alimentación del niño en el primer año de vida. Conferencia transmitida en la hora de la higiene organizada por la Secretaría de Higiene y 
esto con el propósito de cumplir su misión de popularizar las disposiciones médicas alrededor de la maternidad. Los médicos se concibieron como los «abanderado[s] en la marcha secular de las civilizaciones» ${ }^{32}$ destacando la labor social que cumplían para el progreso de la nación.

Producto de esto la maternidad dejó de ser una preocupación exclusiva del mundo femenino para pasar a ser un asunto público ratificando que la mujer era importante en la búsqueda del progreso nacional, así lo afirmaba Alfonso Castro en 1930: «si queremos hombres capaces y patria digna, tenemos que formar primero madres verdaderas ${ }^{33}$. Esta preocupación continúo en la década de 1950, en la cual se siguió presionando por generar medidas de cuidado prenatal ${ }^{34}$. No obstante, los médicos tenían presente la realidad a la que muchas madres obreras se enfrentaban, trabajando «en ocasiones como verdaderas bestias hasta en que el momento el centinela del dolor les anuncia que ya es la hora de la maternidad $\iota^{35}$.

Debido a las condiciones materiales a las que se enfrentaban las mujeres de más escasos recursos se buscó mejorar el acondicionamiento de los servicios sanitarios del país «con el objetivo de disminuir la mortalidad tanto materna como infantil $»^{36}$. Es así, como en Barranquilla se realizaron

Asistencia social del Departamento», Revista de Higiene, $\mathrm{n}^{\circ} 35$ (1944): 5.

32 Dr. Nicolás Dumar, "Día Panamericano de la salud, palabras inaugurales», Revista de Higiene, $\mathrm{n}^{\circ}$ 31-32 (1943): 39.

33 Alfonso Castro, «Un análisis sobre la Higiene en Colombia por el profesor Alfonso Castro», El Tiempo, Bogotá, 18 de junio de 1930, 8. Acceso el 25 de marzo de 2020, https://news.google.com/ newspapers?nid=N2osnxbUuuUC\&dat=19300618\&printsec=frontpage\&hl=es.

34 Jorge Bejarano, Conferencia sobre Puericultura. Higiene del Embarazo (Bogotá: Biblioteca Nacional de Colombia, 1953), [Archivo de audio]. Acceso el 25 de marzo de 2020, https://catalogoenlinea.bibliotecanacional.gov.co/client/es_ES/search/ asset/85541/0.

35 Bejarano, Conferencia sobre..., En países como en Chile se generaron desde principio del siglo XX medidas de protección a la maternidad buscando establecer procesos de protección a la infancia. Ver: Lidia Casas y Ester Valenzuela, «Protección a la maternidad: una historia de tensiones entre los derechos de infancia y los derechos de las trabajadoreas», Revista de Derecho vol. XXV, nº 1 (2012): 77-101.

36 Urdaneta, «la importancia de los...», 12. 
programas de apoyo a la maternidad reflejando medidas que buscaban mejorar las condiciones de gestación, como la ampliación de la capacidad del Pabellón de Maternidad «Leopoldina de Santos». Durante su inauguración, el 3 de junio de 1944, el Diputado Félix E. Barrios manifestaba que este tipo de obras eran parte del deber del Estado, ya que "la madre desvalida, abandonada al peso de su pobreza en el período prenatal, influye con su carácter negativos en la conformación espiritual y física del futuro ciudadano» ${ }^{37}$. Un punto interesante del discurso, es que las políticas de higiene y salubridad del Estado, que buscaban impactar en el proceso de maternidad, estuvieron mediadas por el conocimiento médico-científico.

Otras estrategias implementadas en Barranquilla fueron: un restaurante materno en el barrio Rebolo ${ }^{38}$, dos patronatos infantiles y un consultorio de gota de leche en el barrio San Isidro ${ }^{39}$. Así mismo se envió a un grupo de estudiantes a la ciudad de Bogotá a un curso de protección infantil y materna, mediante una beca brindada por el Departamento del Atlántico ${ }^{40}$. Estas políticas públicas buscaron generar estrategias de intervención en la maternidad y el cuidado del infante.

37 Félix E. Barrios, «En el Hospital de Caridad. Se aumenta la capacidad del pabellón de Maternidad "Leopoldina de Santos" Solemne inauguración de siete modernos departamentos para pensionados», Revista de Higiene, ${ }^{\circ} 34$ (1944): 25.

38 Dr. Luis Enrique Cerra, «Nutrición y Mortalidad Infantil. Charla en la hora de la higiene organizada por la Secretaría de Higiene y Asistencia Social del Departamento", Revista de Higiene, $\mathrm{n}^{\mathbf{0}} 36$ (1944): 32.

39 Para el período de estudio se encontraban en proceso de construcción los patronatos de los municipios de Puerto Colombia y Soledad. La información de los patronatos y la gota de leche es tomada de: «Protección Infantil y Materna», Revista de Higiene, $\mathrm{n}^{\circ} 36$ (1944): 30. Sobre las gotas de leche como estrategía para el cuidado de la infancia ver: Carlos Viesca, "La Gota de leche. De la mirada Médica a la atención médico-social en el México Posrevolucionario», en Curar, Sanar y Educar. Enfermedad y Sociedad en México, siglos XIX y XX, coord. Claudia Agostini (México: Universidad Nacional de México, Benemerita Universidad Autonoma de Puebla, 2008), 195-218. Acceso el 29 de junio de 2020, http://www.historicas.unam.mx/ publicaciones/publicadigital/libros/curar_sanar/494.html.

40 Ordenanza 58/1943, del 16 de junio, por la cual se crean unas becas en la Escuela Nacional Superior de Enfermeras, Revista de Higiene, nº 27-28 (1943): 28. 
Fue así como los médicos trazaron las coordenadas de la mujer en su rol de madre ${ }^{41}$. Para ellos, era la que permitía la vida y la encargada del desarrollo y bienestar del niño, por lo cual centraron su atención en este aspecto ${ }^{42}$. Uno de los elementos a los que mayor centralidad se otorgó fue a la alimentación del infante, especialmente en el primer año de vida debido a que era donde se concentraban las tasas de mortalidad infantil, tópico que abordaremos en las siguientes líneas.

\section{La madre y la alimentación del niño ¿Lactancia o alimentación sustitutiva?}

La nutrición de los niños era un punto clave en que los médicos hicieron bastante énfasis a las madres, debido a que muchas de las enfermedades infantiles que asolaban a esta población se encontraban relacionadas con la alimentación ${ }^{43}$. Fue así como a través de la prensa, conferencias y manuales se incentivó a que las mujeres lactaran a sus hijos, debido a que «una vez comprobada la asepsia de la leche materna, se estimuló la lactancia; la madre que se negaba a hacerlo era considerada despiadada $[y]$ desnaturalizada ${ }^{44}$. Al igual que sucedió con la gestación y el parto, se buscó por medio de la lactancia controlar todas las etapas reproductivas de la mujer, con los cánones y consejos de la comunidad médica difundidos en los medios de comunicación de la época. El objetivo era resaltar los beneficios de la lactancia materna en el desarrollo del niño el cual fue considerado «un ser en construcción permanente y

\footnotetext{
41 Este era el rol ideal asignado a las mujeres. Sin embargo, los médicos del período también vieron con preocupación cómo la prostitución se constituía en un fenómeno que afectaba la salud pública convirtiéndose en un vector de enfermedades. Ver: Angela L. Agudelo, Willian Chapman y Saray Martínez, «La prostitución como un problema de salubridad pública: Prostitutas, violencia y leyes en el Departamento del Atlántico, 1911-1950», Historia Caribe vol. 15, n 37 (2020): 193-213, doi: https:// doi.org/10.15648/hc.37.2020.8.

42 Palomar, «Maternidad: Historia,» 47.

43 Márquez y Gallo, «Eufemismo para,» 21-48.

44 Micolta, «Apuntes históricos de la paternidad...», 103, de esta opinión era el Doctor Ernesto Brando quien afirmaba en 1944 que «toda madre tiene el deber de amamantar a su hijo. No hacerlo no solamente significa que la madre no cumple sus deberes primordiales, sino que el instinto maternal es muy bajo en ella.» Brando, «Alimentación del niño en el primer año de vida,» 9.
} 
continúa, por lo tanto, se le debe aportar todos los elementos necesarios para un buen desarrollo» ${ }^{45}$. La lactancia materna era un componente clave en el desarrollo del infante, por lo cual, en el primer Congreso del Niño, realizado en 1936 en Colombia, se buscó garantizarla como un derecho bajo la responsabilidad materna y social, esta última en ausencia de sus padres debía responder por el proceso alimentario del niño ${ }^{46}$.

El amamantamiento tenía que hacerse en las mejores condiciones de asepsia y salud, con el fin de que la mujer no trasmitiera enfermedades a su prole, por lo que se instaba a conservar correctos hábitos llamando «la atención de la mujer que amamanta [,] de la necesidad indispensable de regular sus cuidados higiénicos en ese período tan precioso para la vida y el porvenir del niño» ${ }^{47}$. Así mismo, se aconsejaba que el infante no podía lactar a libre demanda, sino que a este se le debía regular la alimentación durante cada tres o cuatro horas, con el fin de adaptar el organismo de los niños a la leche materna $^{48}$.

Otras de las razones que tuvieron los médicos para no aconsejar la lactancia a libre demanda era que consideraban que existía una relación entre el exceso de amamantar y la fatiga del cuerpo femenino. Así lo afirmaba el médico Ernesto Brando en 1943:

45 Brando, «Alimentación del niño en el primer año de vida,» 7.

46 Dr. José M. Garrido, "Consideraciones vitales de la Infancia Conferencia del Dr. José M. Garrido, radiodifundida en la "hora de la higiene" organizada por la Secretaría de Higiene y Asistencia Social», Revista de Higiene, n’ 38 (1944): 7.

47 «Nociones de puericultura e higiene infantil para las madres jóvenes de Colombia XIII», El tiempo, Bogotá, 15 de enero 1930, 2. Acceso el 25 de marzo del 2020, https://news.google.com/ newspapers?nid=N2osnxbUuuUC\&dat=19300115\&printsec=frontpage \&hl=es.

48 Este criterio de horarios puede encontrarse en Torres y Vasco, Nociones de Puericultura..., 8; Calisto Torres Umaña, Conferencia sobre Puericultura. La alimentación del niño (Bogotá: Biblioteca Nacional de Colombia, 1953), [Archivo de audio]. Acceso, 25 de marzo de 2020, https://catalogoenlinea.bibliotecanacional.gov.co/ client/es_ES/search/asset/86652/0. Brando, «Alimentación del niño en el primer año de vida,» 7; A Peña Chavarría, «Nociones de Higiene y Puericultura infantil», El Tiempo, Bogotá, 18 de diciembre, 1929 2. Acceso el 25 de marzo de 2020, https://news.google. com/newspapers?nid=N2osnxbUuuUC\&dat=19291218\&printsec=frontpage \&hl=es; Dr. G. Variot, La puericultura, consejos a las madres para la crianza de sus hijos (Bogotá: Tip. Mogollón, 1910), 28. 
Porque la falta de reglas en [el amamantamiento] acarrea quebrantos en la salud materna. Sus glándulas, llamadas a un trabajo intenso estimulan el organismo todo el cual en un principio responde, pero después, como un caballo obligado a una carrera superior a sus fuerzas, cae rendido y desfallecido ${ }^{49}$.

Incluso se afirmaba que, si la madre llegaba a amamantar a sus hijos a libre demanda, esto podría provocar el desarrollo de enfermedades:

La lactancia prolongada mina y destruye considerablemente el organismo de la madre; por abusar de ella muchas mujeres despiertan una tuberculosis que yacía dormida sin mayores síntomas escondida de sospecha medica y que solo un examen radiológico podría haber descubierto ${ }^{50}$.

Además de los horarios establecidos para amamantar, las mujeres debieron establecer una alimentación balanceada indispensable para reducir los índices de mortalidad infantil ${ }^{51}$. Entre los cánones médicos alimenticios para mejorar la lactancia se encontraban ingerir cantidades considerables de agua, leche de origen vacuno e incluso cerveza ${ }^{52}$. En la prensa aparecen también sugerencias que buscaron aumentar la cantidad de leche materna ligadas a los estados de descanso como el reposo y el sueño ${ }^{53}$, los cuales eran muy difíciles de cumplir por parte de las madres obreras, por lo cual se aconsejó que se «instituyeran salas cunas para cuidar en las horas laborales al niño a cargo de una enfermera, con esto se garantizaba las horas de lactancia hacia el amor» ${ }^{54}$.

49 Brando, «Alimentación del niño en el primer año,» 8.

50 A. Peña Chavarría, «Nociones de Puericultura e Higiene Infantil», El Tiempo, Bogotá, 26 de marzo 1930, 12. Acceso el 25 de marzo de 2020, https://news.google.com/ newspapers?nid=N2osnxbUuuUC\&dat $=19300326 \&$ printsec $=$ frontpage\&hl=es. Este mismo criterio que relaciona la lactancia excesiva con la aparición de enfermedades debilitantes se encuentra también en: «Nociones de puericultura,» 2.

51 Cerra, «Nutrición y Mortalidad Infantil,»10.

52 Esta última era aconsejada por Variot que debía ser sin alcohol o ligada con agua. Ver: Variot, La puericultura, consejos a las madres..., 34.

53 A Peña Chavarria, «Nociones de Puericultura e Higiene Infantil», El Tiempo, Bogotá 12 marzo de 1930, 12. Acceso el 25 de marzo del 2020, https://news.google. com/newspapers?nid=N2osnxbUuuUC\&dat=19300312\&printsec=frontpage\&hl=es.

54 Urdaneta, «la importancia de los», 12. En Chile existió desde principios del siglo XX una serie de políticas públicas que desarrollaron la implementación de sala cunas. Ver: Casas y Valenzuela, «Protección a la maternidad,» 80-81. 
Sin embargo, estas lecciones de alimentación e implementación de medidas laborales de apoyo a la maternidad diferían de la realidad encontrada por los médicos. Un ejemplo de esto, lo tenemos en la encuesta difundida por Luis Enrique Cerra, director del Patronato Infantil de Rebolo (Barranquilla), en 1944, donde señalaba que 500 madres que asistían a este centro se encontraban en condición de desnutrición ${ }^{55}$, demostrándolo con la exposición de su régimen alimenticio basado "excesivamente [en] hidrocarbonado deficiente en proteínas que son las sustancias indispensables para el sostenimiento y transformación de la vida celular del organismo» ${ }^{56}$, por lo que manifestaba que: «la desnutrición materna trae como consecuencia lógica la disminución de la lactancia, ciertas enfermedades agudas y crónicas y sobre todo desgraciadas condiciones de la vida obrera imponen la necesidad de la alimentación artificial tan peligrosa, aunque sea considerada por una madre inteligente y comprensiva ${ }^{57}$.

Debido a esto, la alimentación láctea en polvo era otra de las preocupaciones que tenía la comunidad médica barranquillera alrededor de la nutrición infantil; entre los cuestionamientos que hacían estaba el hecho de que muchas mujeres no pudieran lactar a sus hijos: «Todas las hembras de los animales llamados mamíferos, alimentan a sus hijos hasta que la naturaleza no les da el poder que su digestión necesita para utilizar sin peligro otra clase de alimentos. ¿Por qué ha de ser la mujer la única que no tiene esta capacidad?» ${ }^{58}$.

La imposibilidad de lactar implicaba que la madre tuviera que recurrir a sucedáneos para poder brindar alguna alimentación a sus hijos, generando enfermedades en los infantes. Los médicos eran enfáticos al afirmar que «En Barranquilla se mueren más niños matados por maicenas $\mathrm{y}$ féculas que por infecciones intestinales» ${ }^{59}$. A parte del uso de farináceos como reemplazo de la lactancia en la prensa

\footnotetext{
55 Cerra, «Nutrición y Mortalidad Infantil», 10.

56 Cerra, «Nutrición y Mortalidad Infantil», 11.

57 Cerra, «Nutrición y Mortalidad Infantil», 11.

58 Torres y Vasco, Nociones de Puericultura...,9.

59 Dr. Ernesto Brando, "Alimentación del niño en el primer año de vida. Conferencia transmitida en la hora de la higiene organizada por la secretaria de Higiene y Asistencia Social del Departamento [Continuación]», Revista de Higiene, $\mathrm{n}^{\circ} 36$ (1944): 8.
} 
se divulgaba publicidad de alimentos lácteos en polvo como sustitutos de la leche materna (imagen No. 1), que prometían los nutrientes necesarios para el crecimiento de niños «robustos y hermosos» ${ }^{60}$.

La publicidad (imagen $n^{\circ} .1$ ), aseguraba que los infantes tomarían una complexión física denominada "rolliza». No obstante, Calixto Torres Umaña criticaba a las «madres, sobre todo para las que pertenecen a nuestros altos medios sociales, el deseo de gordura en sus hijos tiene tan pocos límites como el deseo de enflaquecerse ellas mismas. Errores a mbos que pueden acarrear consecuencias igualmente funestas. El niño obeso no está en estado normal de nutrición ${ }^{61}$. Así mismo, señalaba que «el único alimento completo, adaptables a la digestión del niño y capaz de llenar sus necesidades nutritivas durante el primer año, es la leche. La leche es, pues, irremplazable» ${ }^{62}$. Otros médicos fueron mucho más críticos con la alimentación láctea en polvo observándola como la causante de enfermedades, apuntando que: «es el niño quien sufre; obligado a tomar un alimento antinatural, su estómago se cansa principia a enflaquecer, de la diarrea sigue peor; dentro de un mes será carne de cementerio» ${ }^{63}$; además, mostraban una inquietante preocupación por el atiborramiento publicitario alrededor de los sustitutos lácteos ya que existían:

[..] en el comercio tal cantidad extraordinaria de leches artificiales que el mismo médico se encuentra perplejo cuando va a escoger algunos de estos productos. Lo peor del caso es que las casas fabricantes con una falta de ética del todo lamentable anuncian al público su producto y, la misma madre ignorante en materia dietética infantil, se ve tentada a cada momento por una propaganda absurda, a cambiar de potes, así como se cambia de vestidos. Cada casa comercial anuncia su producto como el mejor de la plaza y casi llega el descaro de asegurar que puede competir con ventaja con la leche materna ${ }^{64}$.

60 «Glaxo Cría niños robustos hermosos», El tiempo, Bogotá, 23 de abril

de 1930, 13. Acceso, el 25 de marzo del 2020, https://news.google.com/ newspapers?nid=N2osnxbUuuUC\&dat=19300423\&printsec=frontpage\&hl=es.

61 Torres y Vasco, Nociones de Puericultura..., 8.

62 Torres y Vasco, Nociones de Puericultura..., 10-11.

63 Brando "Alimentación del niño en el primer», 8.

64 Brando «Alimentación del niño en el primer», 9. 
Sin embargo, a pesar del rechazo que algunos médicos realizaron a la alimentación láctea en polvo, muchos llegaron a la conclusión que algunas mujeres simplemente no podían amamantar a sus hijos, señalando que esto era producto de: las condiciones materiales de vida, a raíz de que a la «mujer desnutrida [...] no se le puede exigir que amamante a su hijo» ${ }^{65}$ y de las circunstancias psicosociales, producto de «la delicadeza del sistema nervioso de la mujer, la intervención, sobre todo, del elemento intelectual, son sin duda responsable de esta anomalía» ${ }^{66}$.

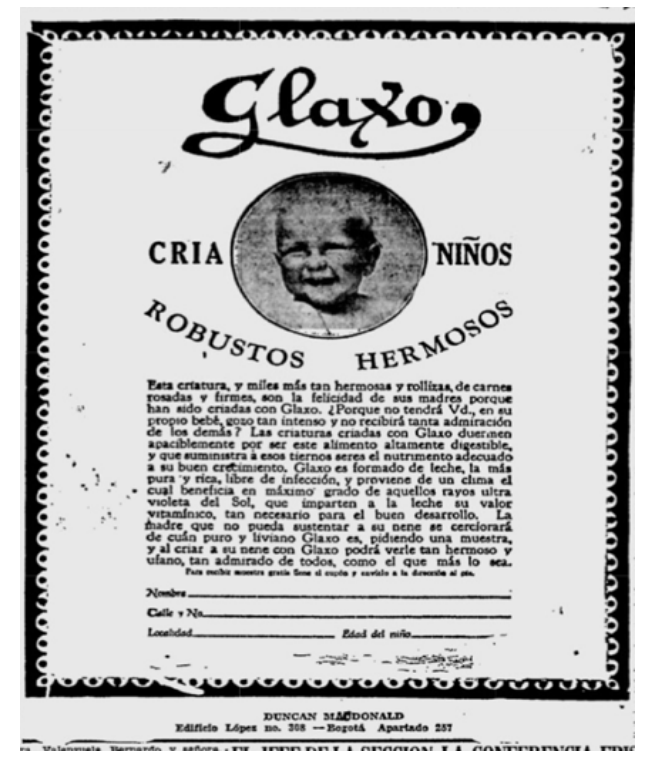

Imagen No. 1 publicidad Glaxo cría niños robustos hermosos

Fuente: El tiempo, Bogotá, 23 de abril de 1930, $13^{67}$.

Aun así, la comunidad médica era enfática en apuntar que cuando la madre no podía amamantar debía recurrir a un sucedáneo. Igualmente, reconocían que productos como Glaxo «son tan caros que solamente las clases acomodadas pueden

65 Cerra, «Nutrición y Mortalidad Infantil», 32.

66 Torres y Vasco, Nociones de Puericultura..., 9-10.

67 El tiempo, Bogotá, 23 de abril de 1930, 13. Acceso, el 25 de marzo del 2020, https:// news.google.com/ newspapers?nid= N2osnxbUuu UC\&dat= 19300423 \&printsec= frontpage $\& h l=e s$. 
servirse de ellos» ${ }^{68}$, por lo cual a las madres obreras y de las clases populares, no les quedaba otra opción que acudir a la leche de origen vacuno, del que se desconfiaba por contener «dos peligros: es el primero, su digestibilidad no es tan fácil como la leche de la mujer, y el segundo estriba en la facilidad de infección ${ }^{69}$.

Este llamado de atención a la salubridad sobre el uso y el papel de la leche de origen vacuno se publicó en la prensa ${ }^{70}$, en los anuncios publicitarios ${ }^{71}$ que enfatizaban en las enfermedades ${ }^{72}$ adquiridas por el consumo de leche adulterada o sin higienizar. Con relación a este hecho, el médico Ernesto Brando, advertía en 1944, que en Barranquilla existía una «mala suministración de la leche de vaca», agregando: "Este producto que, por ser único alimento de los niños tendría que ser sagrado, se presta para toda clase de manipulaciones por parte de la gente criminal que, a costa de ganar unos pocos centavos de más, es capaz de matar a sus hijos» ${ }^{73}$. La comunidad médica de la época desconfiaba de este tipo de sucedáneos para los menores entre 0 y 1 año de edad, por lo que aconsejaban "que la alimentación artificial no puede ni debe ser la básica en la crianza del niño. Se llegará a ella solamente en casos excepcionales y solamente después que un médico graduado lo autorice» ${ }^{74}$.

A pesar de los esfuerzos realizados por los médicos en popularizar sus conocimientos en materia de crianza y alimentación infantil, estos se vieron enfrentados

68 Brando, "Alimentación del niño en el primer [Continuación]», 9.

69 Torres y Vasco, Nociones de Puericultura..., 11.

70 A. Peña Chavarría, «La Higiene de la leche y su valor nutritivo», El Tiempo, Bogotá, 21 de mayo de 1930, 13. Acceso el 25 de marzo del 2020, https://news.google. com/newspapers?nid=N2osnxbUuuUC\&dat=19300521\&printsec=frontpage\&hl=es.

71 Como los que aparecen en la revista de Higiene en el Departamento del Atlántico durante los años de 1943 y 1944.

72 Según la comunidad médica, las enfermedades y gérmenes que se trasmitían a través de la leche de origen vacuno eran: «de la tuberculosis, de la fiebre tifoidea, difteria, disentería, estreptococias y otras que pueden provenir sea del animal, del ordeñador y otros manipuladores.» Brando, "Alimentación del niño en el primer [Continuación],» 6.

73 Brando, «Alimentación del niño en el primer [Continuación]», 6.

74 Brando, «Alimentación del niño en el primer [Continuación]», 9. 
constantemente con el conocimiento empírico acumulado a lo largo de los años por las mujeres, por lo que no era extraño que las abuelas, comadres y hermanas cuestionaran las disposiciones sanitarias. Así se reflejaba en las quejas escritas por Peña Chavarría en 1930 en el periódico El Tiempo, alrededor de las directrices sobre el período de lactancia (un año) ${ }^{75}$ :

[...] más que en ningún otro asunto, en este particular es donde ella pone oídos a los consejos de la abuela, de la comadre, de la tía o de la vecina, que, por su experiencia aislada y personal desde luego, dan indicaciones muchas veces contradictorias y perjudiciales dejando a la madre inexperta en la mayor incertidumbre ${ }^{76}$.

Pero, no fue solo el destete donde la comunidad médica observó con preocupación la injerencia de tías, abuelas y comadres, también en lo relacionado con los sucedáneos de la leche materna. En los medios de comunicación de la época apuntaban:

[...] intervienen otra vez las abuelas, las comadres, las amigas del vecindario y dicen a esta pobre mártir de su deber, ayude al niño con algo. Se reúne el consejo de familia; cada cual expone su parecer y principia con el agua de panela, después con el sagú y, finalmente, se llega a la leche de vaca o a los productos industriales ${ }^{77}$.

Así mismo, señalaban que estos consejos brindados por la tradición femenina perjudicaban el desarrollo infantil. Estas injerencias, las podemos leer como una disputa entre el conocimiento científico abanderado por la comunidad médica y el saber empírico, acumulado por las mujeres a través de largos años de crianza. Este hecho lo percibimos con mayor claridad en el cuestionamiento que hacía el médico Brando:

75 Así mismo en la Columna de Peña de 1930 se afirmaba: «Por ningún motivo debe alimentarse al pecho a un niño sano después de un año» Peña, "Nociones de Higiene,» 12.

76 Peña, «Nociones de Higiene», 12.

77 Brando «Alimentación del niño en el primer», 8. 
La abuela entonces dirá que el médico con todas esas reglas está matando a la pobre criatura que en su tiempo el niño se criaba bien. Y principian consejos de comadres amigas y parientas; con profunda sabiduría nos harán la pregunta siguiente: ¿Cómo nos hemos criados nosotras? Nosotros contestaríamos por pura suerte: porque en otros tiempos ¿cuántos niños no se morían por incontenibles diarreas o infecciones intestinales? ${ }^{78}$.

Es a partir del conocimiento empírico femenino, que a la vez fue desacreditado, que se empezó a construir un discurso médico en torno a la mujer y al ejercicio de su rol materno, por lo que vieron necesario establecer cánones y consejos médicos que otorgaran coordenadas para el desarrollo de la maternidad. Estas ideas desestimaron el papel del instinto materno y el accionar de la madre, señalando que «la mayoría de las madres no sabe criar a sus hijos. Es doloroso decirlo, pero es la pura verdad ${ }^{79}$.

La idea de incapacidad llevó a que las madres fueran medidas en su competencia a partir de los logros obtenidos en el desarrollo infantil. Un niño saludable era producto de una madre responsable. Las mujeres se vieron sometidas a la presión social «al convertir al hijo en el parámetro de su desempeño como "buena madre", entendida a partir de evaluaciones hechas con criterios supuestamente científicos» ${ }^{80}$.

Producto de esto, en Barranquilla se hizo una competencia para las madres de clases populares con el objetivo de mostrar cuál de ellas tenía a su hijo más sano. Este evento fue desarrollado en el día panamericano de la salud, el 2 de diciembre de 1994; y fue titulado "Concurso del niño sano", con sede en el Patronato infantil ${ }^{81}$. El evento fue descrito en la

78 Brando «Alimentación del niño en el primer», 7.

79 Brando, «Alimentación del niño en el primer», 6.

80 Palomar «Maternidad: Historia y cultura», 47.

81 El panel de jurados estuvo conformado por Arturo Ponce Rojas, Secretario de Higiene y Asistencia Social del Departamento del Atlántico, el alcalde de Barranquilla Rafael Borelly, Ernesto Brando, director del Patronato de Protección Infantil y el médico Carlos Urueta Ferrans. Dr. Argos, «Día Panamericano de la salud», Revista de Higiene, $\mathrm{n}^{\circ} 38$ (1944): 19. 


\section{Revista de Higiene del Departamento del Atlántico con estas palabras:}

[...]carácter especial por la presencia de tantas madres y tantas caritas ingenuas y sonrosadas que ponían alegría $\mathrm{y}$ esplendor a tan hermoso espectáculo. Hubo premios y menciones entre los más saludables y el derecho a la mejor asistencia médica del pequeño "campeón" de precaria salud en el Hospital Infantil de San Francisco de Paula ${ }^{82}$.

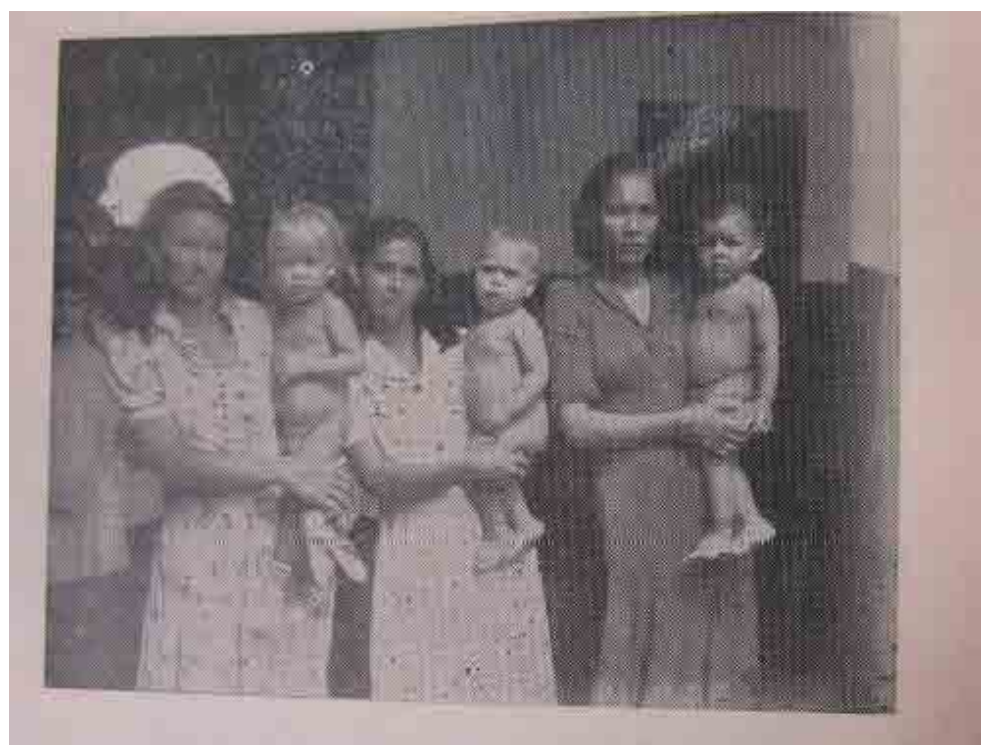

Imagen No. 2 concurso del niño sano-Niños ganadores de primero, segundo y tercer premio en brazos de sus madres ${ }^{83}$.

Fuente: Revista de Higiene, $\mathrm{n}^{\circ} 38$ (1944): 19.

El concurso buscó resaltar el buen resultado que estaban teniendo los Patronatos infantiles en su estrategia de orientar a las madres en el cuidado y nutrición de sus hijos. La revista reprodujo la imagen de las tres madres ganadoras (imagen No. 2), al igual que una variedad de fotografías de los niños galardonados. A partir de esta estrategia se pretendió que las madres colocaran en práctica las directrices médicas relacionadas con la alimentación infantil.

82 Argos, «Día Panamericano», 24- 25.

83 Argos, «Día Panamericano», 22. 


\section{Reflexiones finales}

Hemos resaltado cómo para los médicos el rol de la madre jugó un papel importante para la construcción y fortalecimiento de la nación. Esto se vio reflejado a través de consejos y cánones mediante los cuales los médicos buscaron popularizar sus conocimientos científicos empleando los medios de comunicación, con el objetivo de establecer un modelo de madre que se preocupará por el desarrollo infantil. Este proceso fue mucho más palpable durante la década de los $30 \mathrm{y}$ 40 del siglo XX, donde se encuentra una mayor participación de los médicos en los medios de comunicación como el periódico El Tiempo o creando sus propios medios de difusión como la Revista de Higiene del Departamento del Atlántico.

El proceso de medicalización de la maternidad tuvo una doble configuración: entre más atención tuviera la infancia mayor relevancia recobró la madre como eje de las sociedades del futuro. En este interés por proliferar el saber médico se vieron enfrentados al conocimiento empírico de las abuelas, tías y comadres que disputaban la influencia en el campo de acción sobre la madre y la niñez, por lo cual, los médicos fueron insistentes en resaltar la insalubridad que conllevaba el dar continuidad al saber tradicional femenino.

Por lo cual, los discursos médicos buscaron transformar las prácticas femeninas en torno a la maternidad, la que dejó de ser un asunto privado de interés solo de la familia para convertirse en un asunto público, que fue materia de discusión e interés por parte de la comunidad médica y de las políticas públicas, que buscaron a través de estas disposiciones que la mujer conservara su papel dentro del hogar, justo en el momento en que Colombia atravesaba por importantes cambios en materia de inserción laboral femenina.

A partir de esto, los discursos médicos buscaron transformar las prácticas femeninas alrededor de la alimentación infantil, ya que esta era observada como uno de los factores que podían desencadenar problemas en el desarrollo del niño e incluso la muerte. La alimentación 
infantil fue un tema de relevancia que estuvo presente durante toda la primera mitad del siglo XX. Iniciando con los manuales de puericultura de principios de siglo y finalizando con las conferencias médicas de la década de los 50.

En estas fuentes los médicos abogaron por la leche materna como el alimento por excelencia para el crecimiento de los infantes e instaron a las mujeres para que ejercieran la lactancia, criticando la implementación de sucedáneos. De estos, la leche de origen vacuno, era para los médicos, la que mayores perjuicios ocasionaba al infante debido a su capacidad de trasmitir agentes patógenos que podían afectar la salud. En este sentido, estrategias como los patronatos, los comedores maternos y las gotas de leche buscaron subsanar la problemática alimentaria de la niñez, y junto a los concursos nutricionales, evidenciaron los esfuerzos que realizó la comunidad médica para materializar el discurso centrado en modificar las prácticas alrededor de la maternidad y lactancia.

\section{Referencias}

\section{Fuentes primarias}

Archivo Histórico del Atlántico (AHA), Barranquilla-Colombia Fondo. Colecciones Hemerográficas.

Barrios, Félix E. «En el Hospital de Caridad. Se aumenta la capacidad del pabellón de Maternidad "Leopoldina de Santos" Solemne inauguración de siete modernos departamentos para pensionados». Revista de Higiene, ${ }^{\circ} 34$ (1944): 21- 27.

Bejarano, Jorge. Conferencia sobre Puericultura. Higiene del Embarazo. Bogotá: Biblioteca Nacional de Colombia, 1953. [Archivo de audio]. Acceso el 25 de marzo de 2020. https:// catalogoenlinea.bibliotecanacional.gov.co/client/es_ES/ search/asset/85541/0.

Brando, Ernesto. Dr. "Alimentación del niño en el primer año de vida. Conferencia transmitida en la hora de la higiene organizada por la Secretaría de Higiene y Asistencia Social del Departamento». Revista de Higiene, n 35 (1944): 5-11. 
Brando, Ernesto. Dr. "Alimentación del niño en el primer año de vida. Conferencia transmitida en la hora de la higiene organizada por la Secretaria de Higiene y Asistencia Social del Departamento [Continuación]». Revista de Higiene, $\mathrm{n}^{\circ} 36$ (1944): 5-9.

Castro, Alfonso. «Un análisis sobre la Higiene en Colombia por el profesor Alfonso Castro». El Tiempo, Bogotá, 18 de junio de 1930. Acceso el 25 de marzo de 2020. https://news.google. com /newspapers?nid= N2osnxbUuu UC\&dat=1 9300618 $\&$ printsec $=$ frontpage $\&$ hl $=$ es.

Cerra, Luis Enrique. Dr. «Nutrición y Mortalidad Infantil. Charla en la hora de la higiene organizada por la secretaria de Higiene y asistencia social del Departamento». Revista de Higiene, $\mathrm{n}^{\circ}$ 36 (1944): 9-15-32.

Dr. Argos. «Día Panamericano de la salud». Revista de Higiene, ${ }^{\circ}$ 38 (1944): 17-26.

Dumar, Nicolás. Dr. "Día Panamericano de la salud, palabras inaugurales». Revista de Higiene, ${ }^{\circ}$ 31-32 (1943): 37-42.

«El empleo más importante», El Tiempo, Bogotá, 12 de enero de 1930. Acceso el 3 de febrero de 2020. https://news. google.com/newspapers?nid=N2osnxbUuuUC\&dat= $19300112 \&$ printsec $=$ frontpage $\&$ hl $=$ es.

Garrido, José M. Dr. "Consideraciones vitales de la Infancia Conferencia del Dr. José M. Garrido, radiodifundida en la "hora de la higiene" organizada por la Secretaría de Higiene y Asistencia Social». Revista de Higiene, no 38 (1944): 5-12.

«Higiene y Saneamiento». El Tiempo, Bogotá, 26 de febrero de 1930. Acceso el 05 de febrero 2020. https://news. google.com/newspapers?nid=N2osnxbUuuUC\&dat= $19300226 \&$ printsec $=$ frontpage $\&$ hl=es.

Martínez Sierra, G. "Nuevas cartas a las mujeres: La nueva ley que han de hacer las mujeres». El Tiempo, Bogotá, 5 de enero 1930. Acceso el 05 de febrero de 2020. https:// news.google.com/newspapers?nid=N2osnxbUuuUC\&dat= $19300105 \&$ printsec $=$ frontpage $\&$ hl $=$ es. 
«Nociones de puericultura e higiene infantil para las madres jóvenes de Colombia XIII». El Tiempo, Bogotá, 15 de enero 1930. Acceso el 25 de marzo del 2020, https:// news.google.com/newspapers?nid=N2osnxbUuuUC\&dat $=19300115 \&$ printsec $=$ frontpage $\& \mathrm{hl}=\mathrm{es}$

Pardo, Roberto. Dr. «Importancia del arroz en la alimentación del pueblo, charla del Doctor Roberto Pardo, director del Departamento de nutrición del Servicio Cooperativo Interamericano de Salud Pública». Revista de higiene, $\mathrm{n}^{\circ} 35$ (1944): 28-29.

Peña Chavarría, A. «Nociones de Higiene y Puericultura infantil». El Tiempo, Bogotá, 18 de diciembre, 1929. Acceso el 25 de marzo de 2020. https://news. google.com/newspapers?nid=N2osnxbUuuUC\&dat $=19291218 \&$ printsec $=$ frontpage $\& \mathrm{hl}=\mathrm{es}$.

Peña Chavarría, A. «Nociones de Puericultura e Higiene Infantil». El Tiempo, Bogotá 12 marzo de 1930. Acceso el 25 de marzo de 2020. https://news.google. com $/ \mathrm{n}$ ewspapers? nid = N 2 os nxbUu u C \& dat $=$ $19300312 \&$ printsec $=$ frontpage $\& \mathrm{hl}=\mathrm{es}$.

Peña Chavarría, A. «Nociones de Puericultura e Higiene Infantil». El Tiempo, Bogotá, 26 de marzo de 1930. Acceso el 25 de marzo de 2020. https://news.google. com/newspapers? nid = N 2 os nxbUu u C \& dat $=$ $19300326 \&$ printsec $=$ frontpage $\& h l=e s$.

Peña Chavarría, A. "La Higiene de la leche y su valor nutritivo». El Tiempo, Bogotá, 21 de mayo de 1930. Acceso el 25 de marzo de 2020. https://news. google.com/newspapers?nid=N2osnxbUuuUC\&dat= $19300521 \&$ printsec $=$ frontpage $\&$ hl $=$ es.

Ponce Rojas, Arturo «El fondo de Fomento Municipal y la Asistencia Pública». Revista de Higiene, n² 29-30 (1943): 5-10.

«Protección Infantil y Materna». Revista de Higiene, nº 36 (1944): 30. 
«Ordenanza 58/1943, de 16 de junio, por la cual se crean unas becas en la Escuela Nacional Superior de Enfermeras». Revista de Higiene, $\mathrm{n}^{\circ} 27-28$ (1943): 28.

Torres Umaña, Calisto, y Eduardo Vasco Gutiérrez. Nociones de Puericultura: El cuidado de la salud y la Educación del Carácter. Bogotá: Imprenta Municipal, 1935.

Torres Umaña, Calisto. Conferencia sobre Puericultura. La alimentación del niño. Bogotá: Biblioteca Nacional de Colombia, 1953. [Archivo de audio]. Acceso, 25 de marzo 2020. https://catalogoenlinea.bibliotecanacional.gov.co/client /es_ ES/search/asset/86652/0.

Urdaneta, Luis A. Doctor. "la importancia de los consultorios y de la protección de las mujeres en cinta por el doctor Luis A. Urdaneta». El Tiempo, Bogotá, 22 de enero de 1930. Acceso el 3 de febrero de 2020. https://news. google.com/newspapers?nid=N2osnxbUuuUC\&dat $=19300122 \&$ printsec $=$ frontpage $\&$ hl $=$ es.

Variot, Dr. G. La puericultura, consejos a las madres para la crianza de sus hijos. Bogotá: Tip. Mogollón, 1910.

\section{Bibliográficas}

Agudelo, Ángela L., Willian Chapman, y Saray Martínez. «La prostitución como un problema de salubridad pública: Prostitutas, violencia y leyes en el Departamento del Atlántico, 1911-1950». Historia Caribe vol. 15, n 37 (2020):193-213. Doi: https://doi.org/10.15648/hc.37.2020.8.

Barceló, María Fátima. «Domesticidad, desafío y subversión: la discursividad femenina» Óp. cit. Revista del centro de investigaciones históricas, $\mathrm{n}^{\mathrm{o}} 14$ (2002): 187-212.

Bermúdez, Suzy. El Bello sexo, la mujer y la familia durante el Olimpo radical. Bogotá: Ediciones Uniandes, ECOE ediciones, 1993.

Bud, Robert. Penicillin Triumph and Tragedy. New York: Oxford University Press, 2007. 
Caballero Truyol, Tomás. «Entre los negocios y la familia: Mujer, crédito y sociedad en Barranquilla (Colombia), 1849-1900». Historelo vol. 9, $\mathrm{n}^{\circ} 17$ (2017): 197-220. Doi: http://dx.doi. org/10.15446/historelo.v8n16.53942.

Casas, Lidia, y Ester Valenzuela. «Protección a la maternidad: una historia de tensiones entre los derechos de infancia y los derechos de las trabajadoras». Revista de Derecho vol. XXV, $\mathrm{n}^{\circ}$ 1 (2012): 77-101.

Cott, Nancy F. «Mujer moderna, estilo norteamericano: los años veinte». En Historia de las mujeres. El siglo XX, dirigido por George Duby y Michelle Perrot, 107-124. Barcelona: Taurus 1993.

Gallo, Oscar, y Jorge Márquez. «La mortalidad infantil y la medicalización de la infancia. El caso de Titiribí, Antioquia, 1910-1950». Memoria y Sociedad, nº 20 (2011): 57-89.

Márquez Valderrama, Jorge. Ciudad, miasmas y microbios. La irrupción de la ciencia pasteriana en Antioquia. Medellín: Universidad de Antioquia, 2005.

Márquez, Jorge, y Oscar Gallo. «Eufemismos para el hambre: saber médico, mortalidad infantil y desnutrición en Colombia, 18881940». Historia y Sociedad, no 32 (2017): 21-48. Doi: https:// doi.org/10.15446/hys.n32.55508.

Micolta, Amparo. "Apuntes históricos de la paternidad y la maternidad». Prospectiva, n 13 (2008): 89-121.

Montenegro, Santiago. El arduo tránsito hacia la modernidad: historia de la industria textil colombiana durante la primera mitad del siglo XX. Medellín: Universidad de Antioquia; Norma; CEDE, 2002.

Morales, Jessica. «Salubridad pública en Barranquilla en la década de los treinta del siglo XX: principales causas de muerte y su incidencia con los hábitos de vida». Tesis de pregrado, Universidad del Atlántico, 2013.

Palomar, Cristina. «Maternidad: Historia y cultura». La ventana, $\mathrm{n}^{\circ}$ 22 (2005): 35-66.

Reyes, Ana Catalina. «Mujeres y trabajo en Antioquia durante la primera mitad del siglo XX». En Mujer, nación, identidad y 
ciudadanía: siglos XIX y XX. IX Cátedra Anual de Historia Ernesto Restrepo Tirado (28 al 30 de octubre de 2004), editado por Ana María Díaz Granados, 162-192. Bogotá: Museo Nacional, 2005.

Sánchez, Ramón y otros, «Medicalización de la vida (I)». Rev. Clin Med Fam vol. 4, n² 2 (2011): 150-161.

Santos, Daniela. «Faldas por pantalones: las obreras, la brecha salarial y el sindicalismo femenino en la industria en Colombia en 1945». Documentos CEDE, nº 49 (2017): 1-42.

Suárez, Marcela. «La familia Burguesa y la mujer mexicana en los inicios del siglo XX». En Mujeres latinoamericanas del siglo XX. Historia y cultura Tomo II. Luisa Campuzano, 293-300. México: Universidad Autónoma metropolitana, Casa de las Américas, 1998.

Urteaga, Luis. «Higienismo y ambientalismo en la medicina decimonónica». Acta Hispánica ad Medicinae Scientiarumque Historiam Illustrandam vol. 5-6, (1985): 417-425.

Viesca, Carlos. «La Gota de leche. De la mirada Médica a la atención médico-social en el México Posrevolucionario». En Curar, Sanar y Educar. Enfermedad y Sociedad en México, siglos XIX y XX, coordinado por Claudia Agostini, 195-218. México: Universidad Nacional de México, Benemérita Universidad Autónoma de Puebla, 2008. Acceso el 29 de junio de 2020. http://www.historicas.unam.mx/publicaciones/publicadigital/ libros/curar_sanar/494.html.

Vos Obeso, Rafaela. Mujer, cultura y sociedad: Barranquilla, 19001930. Santa Fe de Bogotá: Fondo de Publicaciones de la Universidad del Atlántico, 1999.

\section{Citar este artículo}

Agudelo-González, Ángela Lucía, y Willian Alfredo ChapmanQuevedo. «Maternidad y lactancia a través del discurso de la comunidad médica en Barranquilla, primera mitad del siglo XX». Historia Y MEMORIA, n 23 (2021): 197-225. Doi: https:/ doi.org/10.19053/20275137.n23.2021.11720. 